\title{
Proximal Interphalangeal Joint 5
}

National Cancer Institute

\section{Source}

National Cancer Institute. Proximal Interphalangeal Joint 5. NCI Thesaurus. Code C102335.

A ginglymoid (hinge) synovial joint within the fifth digit of the hand or foot connecting the proximal and middle phalanges. 